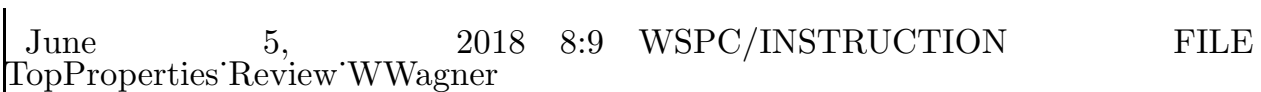

Modern Physics Letters A

(c) World Scientific Publishing Company

\title{
TOP-ANTITOP-QUARK PRODUCTION AND DECAY PROPERTIES AT THE TEVATRON
}

\author{
WOLFGANG WAGNER \\ Fachgruppe Physik, Bergische Universität Wuppertal, Gaußstraße 20, \\ 42119 Wuppertal, Germany \\ wagner@physik.uni-wuppertal.de \\ CDF/PHYS/TOP/PUBLIC/10107 \\ Version 2.0 \\ FERMILAB-PUB-10-056-E \\ June 5,2018
}

\begin{abstract}
At the Tevatron, the collider experiments CDF and $\mathrm{D} \varnothing$ have data sets at their disposal that comprise a few thousand reconstructed top-antitop-quark pairs and allow for precision measurements of the cross section as well as production and decay properties. Besides comparing the measurements to standard model predictions, these data sets open a window to physics beyond the standard model. Dedicated analyses look for new heavy gauge bosons, fourth generation quarks, and flavor-changing neutral currents. In this mini-review the current status of these measurements is summarized.
\end{abstract}

Keywords: Top quark, hadron collider.

PACS Nos.: 14.65.Ha

\section{Introduction}

The top quark is by far the heaviest elementary particle observed by particle physics experiments and features a mass of $m_{t}=173.1 \pm 1.3 \mathrm{GeV} / c^{2}$ [1] The large mass of the top quark gives rise to large radiative corrections, for example to the $W$ propagator, which causes a strong correlation between $m_{W}, m_{t}$, and the Higgs boson mass $m_{H}$. To predict $m_{H}$ a precise measurement of $m_{t}$ is crucial. The large mass leads also to a very short lifetime of the top quark, $\tau_{t} \simeq 0.5 \cdot 10^{-24} \mathrm{~s}$, such that top hadrons are not formed. The top quark thus offers the unique possibility to study a quasi-free quark and as a consequence polarization effects are accessible in the angular distributions of top-quark decay products. Since $m_{t}$ is close to the energy scale at which the electroweak gauge symmetry breaks down (vacuum expectation value of the Higgs field $v=246 \mathrm{GeV}$ ), it has been argued that the top quark may be part of a special dynamics causing the break down of the electroweak gauge symmetry 2. Finally, the top quark gives access to the highest energy scales and offers thereby the chance to find new, unexpected physics, for example heavy resonances that decay into $t \bar{t}$ 


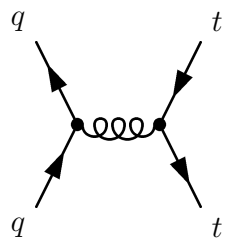

(a)

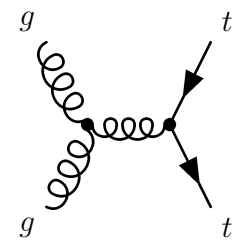

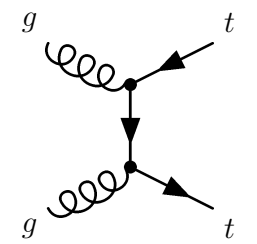

(b)

Fig. 1. Feynman diagrams of the leading order processes for $t \bar{t}$ production: (a) quark-antiquark annihilation $(q \bar{q} \rightarrow t \bar{t})$ and (b) gluon-gluon fusion $(g g \rightarrow t \bar{t})$.

pairs.

In the past years the Fermilab Tevatron, a synchrotron colliding protons and antiprotons at a center-of-mass energy of $\sqrt{s}=1.96 \mathrm{TeV}$, was the only place to produce and observe top quarks under laboratory conditions. Physics data taking of Tevatron Run II started in 2002 and in the meanwhile the accelerator has delivered collisions corresponding to an integrated luminosity of $8.0 \mathrm{fb}^{-1}$. The two general-purpose detectors $\mathrm{CDF}$ and $\mathrm{D} \varnothing$ have recorded collision data corresponding to $6.5 \mathrm{fb}^{-1}$ and $7.0 \mathrm{fb}^{-1}$, respectively.

\section{Top-Antitop Production Cross Section}

The main source of top quarks at the Tevatron is the pair production via the strong interaction. At leading order in perturbation theory $\left(\alpha_{s}^{2}\right)$ there are two processes that contribute to $t \bar{t}$ production, quark-antiquark annihilation $q \bar{q} \rightarrow t \bar{t}$ and gluongluon fusion $g g \rightarrow t \bar{t}$. The corresponding Feynman diagrams for these processes are depicted in Fig. 1.

According to the standard model (SM) top quarks decay with a branching ratio of nearly $100 \%$ to a bottom quark and a $W$ boson and the $t \bar{t}$ final states can be classified according to the decay modes of the $W$ bosons. The most important (or golden) channel is the so-called lepton + jets channel where one $W$ boson decays leptonically into a charged lepton (electron or muon) plus a neutrino, while the second $W$ boson decays into jets. The lepton+jets channel features a large branching ratio of about $29 \%$, manageable backgrounds, and allows for the full reconstruction of the event kinematics. Other accessible channels are the dilepton channel, in which both $W$ bosons decay to leptons, and the all-hadronic channel, where both $W$ bosons decay hadronically. The dilepton channel has the advantage of having a low background, but suffers on the other hand from a lower branching fraction (5\%) compared to the lepton+jets channel. The all-hadronic channel, on the contrary, has the largest branching ratio of all $t \bar{t}$ event categories (46\%), but has the drawback of a huge QCD multijet background, that has to be controlled experimentally. The different categories of $t \bar{t}$ and their branching fractions are summarized in Table 1 . 
Table 1. Categories of $t \bar{t}$ events and their branching fractions. The sum of all fractions is above $100 \%$ because of rounding effects.

\begin{tabular}{cccc}
\hline$W$ decays & $e / \mu \nu$ & $\tau \nu$ & $q \bar{q}$ \\
\hline$e / \mu \nu$ & $5 \%$ & $5 \%$ & $29 \%$ \\
$\tau \nu$ & - & $1 \%$ & $15 \%$ \\
$q \bar{q}$ & - & - & $46 \%$ \\
\hline
\end{tabular}

\subsection{Lepton+Jets Channel}

The experimental signature of lepton + jets $t \bar{t}$ events comprises a reconstructed isolated lepton candidate, large missing transverse energy $\left(E_{\mathrm{T}}\right)$ and at least four jets with large transverse energy $E_{T} \equiv E \cdot \sin \theta$. Two jets originate from $b$-quarks. Typical selection cuts ask for a charged lepton with $p_{T}>20 \mathrm{GeV} / c, E_{\mathrm{T}}>20 \mathrm{GeV}$, and at least four jets with $E_{T}>20 \mathrm{GeV}$ and $|\eta|<2.0$, one of them identified as a $b$-quark jet. The most commonly used algorithm to identify $b$-quark jets is based on the reconstruction of secondary vertices in jets, exploiting the relatively long lifetime of $b$-hadrons and a large Lorentz boost. The typical decay length of $b$-hadrons in high- $p_{T} b$-quark jets is on the order of a few millimeters. The requirement of a secondary vertex within one of the jets leads to a large reduction of the $W+$ jets background by roughly a factor of 50 , while the selection efficiency for $t \bar{t}$ events is about $50 \%$ to $60 \%$. In Run I and Run II several analyses used secondary vertex $b$-jet

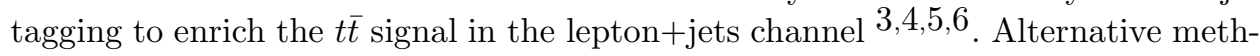
ods identify $b$-quark jets by relying on the impact parameter significance of tracks associated to jets $\frac{718}{8}$, or by reconstructing leptons originating from semileptonic decays of $b$ hadrons 910 . Advanced $b$-jet taggers combine all available information using neural networks 1112 .

The most recent CDF analysis based on secondary vertex $b$ tagging 13 is a counting experiment in which the background rate is estimated using a combination of simulated events and data driven methods. The signal region is defined as the data set with a leptonic $W$ candidate plus $\geq 3$ jets. To further suppress background, a cut on the sum of all transverse energies $H_{T}>230 \mathrm{GeV}$ is applied. The jet multiplicity distribution of the $W+$ jets data set observed by this CDF analysis is shown in Figure 2. The uncertainty on the luminosity measurement is reduced by measuring the ratio of $t \bar{t}$-to- $Z$-boson cross sections and the measured cross section is found to be $7.32 \pm 0.36$ (stat) \pm 0.59 (syst) \pm 0.14 (Z theory) pb, assuming $m_{t}=172.5 \mathrm{GeV} / c^{2}$. The cross section of $t \bar{t}$ production and $Z / \gamma^{*} \rightarrow \ell^{+} \ell$ - production are measured in data samples corresponding to the same integrated luminosity. By forming the ratio of both measured cross sections and multiplying by the well-known theoretical $Z / \gamma^{*} \rightarrow \ell^{+} \ell-$ cross section the luminosity uncertainty of $6 \%$ is effectively removed and replaced by the uncertainty on the $Z / \gamma^{*}$ cross section of $2 \%$.

The identification of $b$-quark jets is a powerful tool to remove $W+$ jets back- 


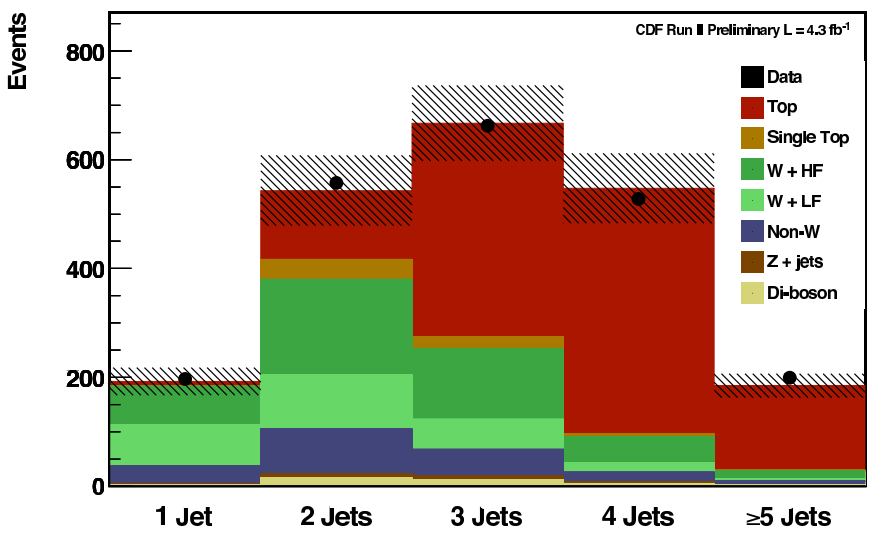

Fig. 2. Jet multiplicity distribution for the $W+$ jets data set, where the $W$ boson is reconstructed in its leptonic decay $W^{ \pm} \rightarrow \ell^{ \pm} \nu_{\ell}\left(\bar{\nu}_{\ell}\right)$. A cut on $H_{T}>230 \mathrm{GeV}$ was applied. The analyzed data set corresponds to $4.3 \mathrm{fb}^{-1}$.

ground. However, the technique is also associated to systematic uncertainties which become relevant when the data statistics increases. The uncertainties are associated to the efficiencies to identify $b$-quark jets, but also to the efficiencies to wrongly identify $c$-quark jets and light-quark jets as $b$ jets. An additional uncertainty arises from the flavor composition of the $W+$ jets data set before applying the tagging algorithm. At $\mathrm{CDF}$, the fraction of heavy-flavor jets is found to be higher in collision data than predicted by the Monte Carlo generator ALPGEN 14 when studying the $W+1$ jets data set using a neural-network-based flavor separating tool 15/16/17. As a result, the fraction of $W b \bar{b}$ and $W c \bar{c}$ is scaled up by a common factor of $1.4 \pm 0.4$. The large uncertainty is assigned to cover the results of studies in higher jet-multiplicity samples and the results obtained by investigating other flavor-separating variables.

An alternative to $b$-quark jet identification is to enhance the $t \bar{t}$ fraction in the candidate sample by exploiting topological or kinematic features of $t \bar{t}$ events. These techniques have also been used extensively at the Tevatron $18|19| 20$.

The single most precise measurement of the $t \bar{t}$ cross section at CDF is based on a neural network technique applied to the $W+\geq 3$ jets data set 21. This method has the advantage that it does not use $b$-quark jet tagging and therefore avoids the systematic uncertainties associated to the $b$-tagging efficiency and the fraction of heavy-flavor jets in $W+$ jets events. The neural network measurement finds a $t \bar{t}$ cross section of $7.82 \pm 0.38$ (stat) \pm 0.37 (syst) \pm 0.15 (Z theory) pb (at $m_{t}=172.5 \mathrm{GeV} / c^{2}$ ).

\subsection{Dilepton Channel}

The final state in which both $W$ bosons originating from the top quark decay either into $e^{-} \bar{\nu}_{e}$ or $\mu^{-} \bar{\nu}_{\mu}$ is called dilepton channel. It features two high- $p_{T}$, isolated charged leptons and large missing transverse energy due to the undetected neutrinos. Final states with $\tau$ leptons are generally not explicitly reconstructed in this channel, 


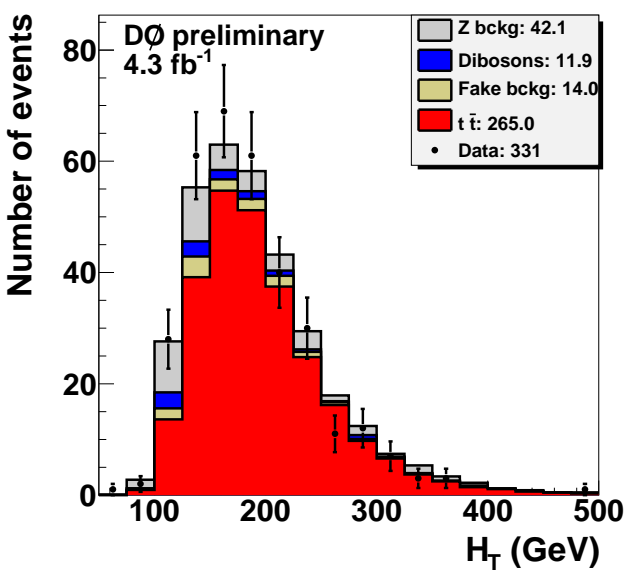

Fig. 3. Distribution of the variable $H_{T}$ for lepton+jets events of collision data corresponding to $4.3 \mathrm{fb}^{-1}$ of integrated luminosity recorded from June 2006 to June 2009.

but contribute indirectly if the $\tau$ decays leptonically into an electron or muon plus neutrinos.

Measurements in the dilepton channel contributed to the discovery of the top quark in the 1990s 22|23|24. In Run II, CDF made several cross section measurements in the dilepton channel 25|26|27. While the standard approach requires both leptons to be well identified as either electrons or muons, analysts at CDF developed a second technique that allows one of the leptons to be measured only as a high- $p_{\mathrm{T}}$ isolated track, thereby significantly increasing the lepton detection efficiency at the cost of a moderate increase in the expected backgrounds.

The most recent measurement by the $\mathrm{CDF}$ collaboration uses a data set corresponding to $4.5 \mathrm{fb}^{-1}$ and is based on events featuring a pair of oppositely charged isolated leptons with $p_{T} \geq 20 \mathrm{GeV} / c, \mathbb{E}_{\mathrm{T}} \geq 25 \mathrm{GeV}$, and two or more jets with $E_{T} \geq 30 \mathrm{GeV}$. The number of selected candidate events is 215 over an expected background of $66.9 \pm 5.7$, yielding a measured cross section of $6.56 \pm 0.65$ (stat) \pm 0.41 (syst) \pm 0.38 (lumi) pb 28 at $m_{t}=175 \mathrm{GeV} / c^{2}$.

At the beginning of Run II, DØ first used fully identified electrons and muons for the dilepton $t \bar{t}$ cross section measurement 29 , but added later also a lepton+track event category 30 . In a dilepton measurement with $1 \mathrm{fb}^{-1}$ of collision data the $\mathrm{D} \varnothing$ collaboration used $e \tau$ and $\mu \tau$ final states in addition to the standard decay channels 31. The $\tau$ leptons are identified in their hadronic decay mode as a narrow jet with low track multiplicity and the identification is optimized using neural networks. The results of this measurement are combined with the most recent analysis of dilepton final states using an integrated luminosity of $4.3 \mathrm{fb}^{-1}$. Fig. 3 shows the distribution of the kinematic variable $H_{T}$ which is defined as the scalar sum of the transverse momenta of the charged lepton and the jets in the event. The resulting 
cross section is $8.4 \pm 0.5$ (stat) ${ }_{-0.8}^{+0.9}$ (syst) $)_{-0.6}^{+0.7}$ (lumi) pb for $m_{t}=172.5 \mathrm{GeV} / c^{2} \underline{32}$.

\subsection{All-Hadronic Channel}

Despite the large branching ratio it is very challenging to isolate a $t \bar{t}$ signal in the all-hadronic channel because of the overwhelming QCD multijet background. Given the limited energy resolution of jets the reconstruction of the $t \bar{t}$ kinematics is also very demanding. The cross section measurements in the all-hadronic channel therefore exploit in most cases topological or event shape information to discern signal from background. The observation of $t \bar{t}$ events in the all-hadronic channel dates back to Run I of the Tevatron 3334 . While CDF used $b$-quark jet identification to suppress background, the $\mathrm{D} \emptyset$ analyses employed a neural network to combine several kinematic variables to one discriminant that separates QCD multijet background from $t \bar{t}$ events.

In Run II, CDF first published an analysis combining $b$-quark jet identification with cuts on several topological variables 35, but replaced these cuts in an updated measurement by a kinematical selection using a neural network 36 . The latest measurement in this series 37 added input variables to the neural network that discriminate between quark-initiated and gluon-initiated jets. As an another novelty the analysis applied techniques used for the measurement of $m_{t}$, namely the simultaneous determination of the top-quark mass $m_{t}$, the jet energy scale, and the number of signal and background events. After the neural network based event selection the signal yields are obtained by a fit to the reconstructed top-quark mass distribution $m_{t}^{\text {rec }}$ and the mass distribution of the $W$ boson candidates. The resulting value for the $t \bar{t}$ cross section is $7.2 \pm 0.5$ (stat) \pm 1.0 (syst) \pm 0.4 (lumi) pb at $m_{t}=172.5 \mathrm{GeV} / c^{2}$.

In its first analysis of Run II the DØ collaboration used a combination of secondary vertex tagging of $b$-quark jets and a neural network to separate $t \bar{t}$ events from QCD multijet events $\frac{38}{38}$. In an updated analysis D $\varnothing$ used an advanced $b$-tagger based on neural networks that features a tagging efficiency of $(57 \pm 2) \%$ for $b$-quark jets and a misidentification rate of $(0.57 \pm 0.07) \%$. At least two jets are required to be tagged in this way. Kinematic information of the events is exploited by combining several variables to a likelihood-ratio discriminant. The measured cross section

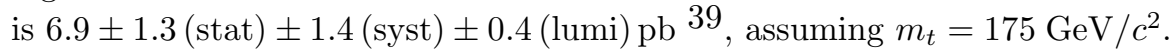

\subsection{Cross Section Combination}

The measurements of the $t \bar{t}$ cross section in $p \bar{p}$ collisions at $\sqrt{s}=1.8 \mathrm{TeV}$ in Run I were combined experiment wise and gave $6.5_{-1.4}^{+1.7} \mathrm{pb}$ at $\mathrm{CDF} 40$ (measured at $\left.m_{t}=175 \mathrm{GeV} / c^{2}\right)$ and $5.7 \pm 1.6 \mathrm{pb}$ at $\mathrm{D} \varnothing \underline{41}\left(m_{t}=172.1 \mathrm{GeV} / c^{2}\right)$. The predicted $t \bar{t}$ cross section at $\sqrt{s}=1.8 \mathrm{TeV}$ is $5.24 \pm 0.31 \mathrm{pb}\left(\right.$ at $\left.m_{t}=175 \mathrm{GeV} / c^{2}\right) 42$.

A summary of the $t \bar{t}$ cross section measurements at CDF in Run II is given in Fig. $4(\mathrm{a})$. The combination of these measurements yields $7.50 \pm 0.31$ (stat) \pm 


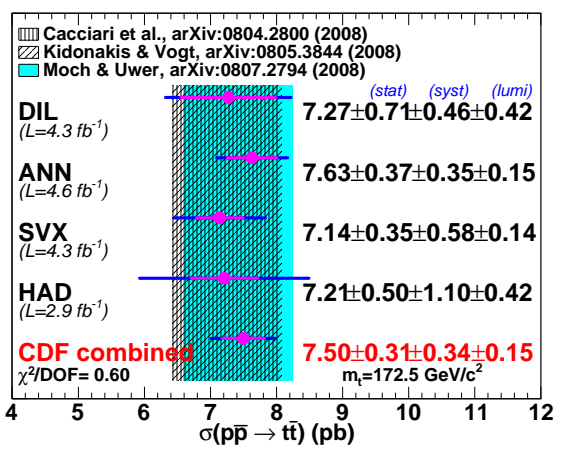

(a)

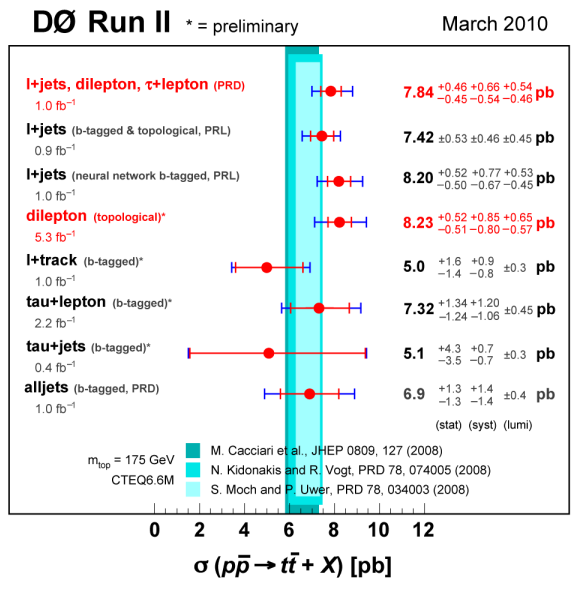

(b)

Fig. 4. Summary of the $t \bar{t}$ cross sections measured by (a) CDF and (b) D $\varnothing$ compared to theoretical predictions.

0.34 (syst) \pm 0.15 (Z theory) pb 43 . The Run II cross section results of the $\mathrm{D} \varnothing$ collaboration are given in Fig. [4](b) The combined cross section is found to be $8.18_{-0.87}^{+0.98} \mathrm{pb}$ (at $m_{t}=170 \mathrm{GeV} / c^{2}$ ) 44 . All measurements are in excellent agreement with theory predictions based on the $\mathrm{SM} 45\left[46 / 47\right.$ which give, for example, $6.90_{-0.64}^{+0.46} \mathrm{pb}$ at $m_{t}=175 \mathrm{GeV} / c^{2}$ 뇨.

\section{Production Properties}

The measured top-quark mass 1 agrees very well with the one predicted by the SM using electroweak precision measurements as input 49 . Also the top-quark pair production cross section is in excellent agreement with the theoretical expectation. Nevertheless, it is important to also test other predictions made by the SM about the top quark to firmly establish its identity.

\subsection{Production Mechanism}

Calculations in perturbative QCD predict that the dominating subprocess of $t \bar{t}$ pair production at the Tevatron is $q \bar{q}$ annihilation (85\%), while gluon-gluon fusion contributes $15 \%$. To measure the fraction of $t \bar{t}$ pairs originating from a $g g$ initial state, physicists at CDF exploited the fact that $g g$ initial states produce more initialstate radiation than $q \bar{q}$ initial states. The analysis makes use of the proportionality of the mean number of low- $p_{\mathrm{T}}$ tracks in an event $\bar{N}_{\text {trk }}$ and the gluon content. The linear relation between $\bar{N}_{\text {trk }}$ and the average number of hard initial-state gluons is calibrated in $W+$ jets and dijet data samples. Using simulated events, templates of the $\bar{N}_{\text {trk }}$ distribution are calculated for $g g \rightarrow t \bar{t}$ and $q \bar{q} \rightarrow t \bar{t}$ events. These templates 
are fit to the distribution observed in collision data, resulting in a measurement of $\sigma(g g \rightarrow t \bar{t}) / \sigma(q \bar{q} \rightarrow t \bar{t})=0.07 \pm 0.14$ (stat.) \pm 0.07 (syst.) 50 . An alternative method exploits the spin information in the top-decay products employing neural networks and sets an upper limit on the $g g$ initiated fraction of $t \bar{t}$ events of 0.61 at the $95 \%$ confidence level (C.L.) 51 .

Recently, the CDF collaboration measured the $g g$-fusion fraction in the $t \bar{t}$ dilepton channel. The measurement exploits the fact that $t \bar{t}$ pairs produced via $g g$ fusion are in a different spin state, namely $J=0, J_{z}=0$, than $t \bar{t}$ pairs produced via $q \bar{q}$ annihilation $\left(J=1, J_{z}= \pm 1\right)$. The different spin state manifests itself in different azimuthal angular correlations of the charged leptons in $t \bar{t}$ dilepton events. Based on a data set corresponding to an integrated luminosity of $2 \mathrm{fb}^{-1}$ a fit to the distribution of the difference $\Delta \phi$ of the azimuthal angles of the charged leptons yields a $g g$-fusion fraction of $f_{g g}=0.53_{-0.38}^{+0.36} 52$. The spin correlation of $t \bar{t}$ pairs was further investigated in a recent measurement based on an integrated luminosity of $4.3 \mathrm{fb}^{-1} 53$. Using lepton+jets events the fraction $f_{o}$ of $t \bar{t}$ pairs in the opposite helicity state $(J=1)$ was determined. The spin correlation can be expressed by the correlation coefficient $\kappa$ which related to $f_{o}$ by $f_{o}=\frac{1}{2}(1+\kappa)$ and found to be $\kappa=0.60 \pm 0.50$ (stat) \pm 0.16 (syst) which is in good agreement with theoretical calculations 54155 .

\subsection{Forward-Backward Asymmetry}

Due to interference effects at next-to-leading order (NLO) QCD predicts a forwardbackward asymmetry

$$
A_{\mathrm{FB}}=\frac{N_{t}(p)-N_{\bar{t}}(p)}{N_{t}(p)+N_{\bar{t}}(p)}=(5.0 \pm 1.5) \%
$$

at the Tevatron $56 \mid 57 / 58$, where $N_{t}(p)$ is the number of top quarks moving in proton direction and $N_{\bar{t}}(p)$ is the number of antitop quarks moving in proton direction. The theoretical uncertainty is driven by the size of higher order corrections to $A_{\mathrm{FB}} 5960$. The asymmetry indicates that top quarks are more likely to be produced in proton direction, while antitop quarks are more likely to be produced in antiproton direction. The relatively small value of $A_{\mathrm{FB}}$ in the $\mathrm{SM}$ is a net result of a positive asymmetry from the interference of the Born amplitude with virtual box corrections ( $t \bar{t}$ final state) and a negative asymmetry from the interference of initial and final state radiation amplitudes ( $t \bar{t} g$ final state). While the $\mathrm{SM}$ value of $A_{\mathrm{FB}}$ is barely measurable at the Tevatron, the measurement is sensitive to non-standard model effects that can reach the size of up to $\pm 30 \%$, e.g., in models with $Z^{\prime}$-like states and parity violating couplings 61 or theories with chiral gluons 57 .

Using events of the lepton+jets topology $\mathrm{CDF}$ and $\mathrm{D} \varnothing$ have investigated the charge asymmetry. In the CDF analysis the hadronic top quark is reconstructed 


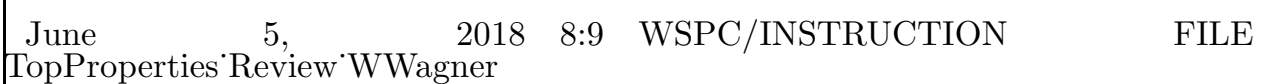

Top-Quark Cross Section and Properties at the Tevatron 9

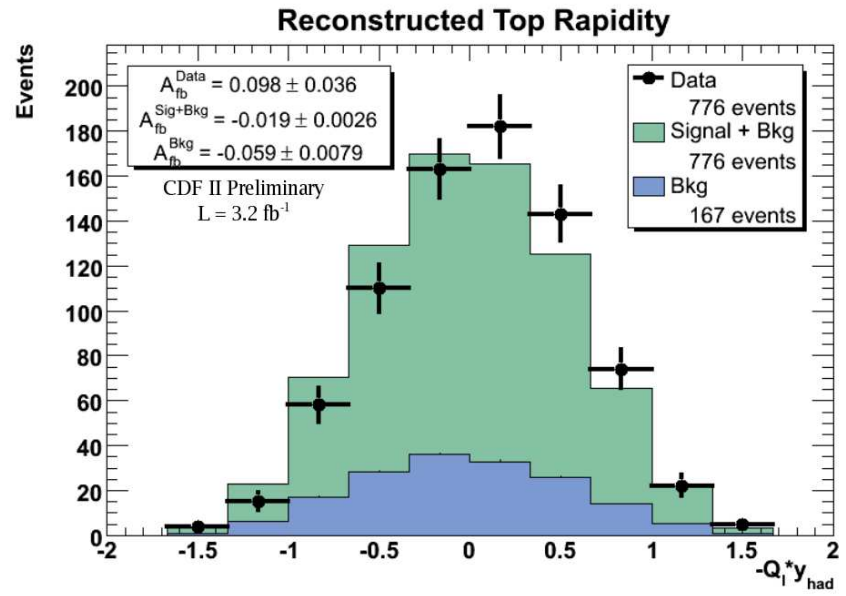

Fig. 5. Distribution of $Q_{\ell} \cdot y_{\text {had }}$ observed by the CDF collaboration in collision data corresponding to an integrated luminosity of $3.2 \mathrm{fb}^{-1}$. The displayed distribution is not corrected for acceptance and reconstruction effects, while these corrections are applied to obtain the measured value of $A_{\mathrm{FB}}$.

and the asymmetry

$A_{\mathrm{FB}}^{\mathrm{lab}}=\frac{N\left(-Q_{\ell} \cdot y_{\mathrm{had}}>0\right)-N\left(-Q_{\ell} \cdot y_{\mathrm{had}}<0\right)}{N\left(-Q_{\ell} \cdot y_{\mathrm{had}}>0\right)+N\left(-Q_{\ell} \cdot y_{\mathrm{had}}<0\right)}=0.193 \pm 0.065$ (stat.) \pm 0.024 (sys.)

is measured 62 , where $Q_{\ell}$ is the charge of the lepton and $y_{\text {had }}$ is the rapidity of the reconstructed hadronically decaying top quark. The CDF measurement quoted in (2) is corrected for background contributions, acceptance bias, and migration effects due to the reconstruction, which has the important advantage that the measured quantity can be directly compared to the theoretically expected one in (11). The relatively large value compared to the SM expectation confirms earlier CDF measurements 64 and has a significance of about two Gaussian standard deviations. The raw $Q_{\ell} \cdot y_{\text {had }}$ distribution before the corrections are applied is shown in Fig. 囵,

$\mathrm{D} \varnothing$ uses $\Delta y \equiv y_{t}-y_{\bar{t}}$ as an observable, applies a background correction and obtains $A=0.12 \pm 0.08 \pm 0.0163$. To compare this value with the CDF measurements or with the theory prediction it has to be corrected for acceptance and migration effects. A prescription for this procedure is provided in ref. 63 . Based on this measurement the $\mathrm{D} \emptyset$ collaboration derives limits on a heavy $Z^{\prime}$ boson that decays to $t \bar{t}$ pairs.

\subsection{Top-Antitop Resonances}

The $t \bar{t}$ candidate samples offer another possibility to search for a narrow-width resonance $X^{0}$ decaying into $t \bar{t}$ pairs by investigating the $t \bar{t}$ invariant mass 65166 . In an analysis using data corresponding to $3.6 \mathrm{fb}^{-1}$ the $\mathrm{D} \varnothing$ collaboration found 
no evidence for such a resonance and places upper limits on $\sigma_{X} \cdot \operatorname{BR}\left(X^{0} \rightarrow t \bar{t}\right)$ ranging from $1.0 \mathrm{pb}$ at $M_{X}=350 \mathrm{GeV} / c^{2}$ to $0.16 \mathrm{pb}$ at $M_{X}=1000 \mathrm{GeV} / c^{2}$ 67. If interpreted in the frame of a topcolor-assisted technicolor model these limits can be used to derive mass limits on a narrow lepto-phobic $Z^{\prime}: M\left(Z^{\prime}\right)>820 \mathrm{GeV} / c^{2}$ at the $95 \%$ C.L., assuming $\Gamma\left(Z^{\prime}\right)=0.012 M\left(Z^{\prime}\right)$. A similar analysis in the all-hadronic channel at CDF yields slightly lower mass limits 68 . Analysts at CDF have also searched for massive gluonic states in the $t \bar{t}$ invariant mass spectrum, setting limits for masses between 400 and $800 \mathrm{GeV} / c^{2} \underline{69}$.

\subsection{Direct Searches for Fourth Generation Quarks}

A straight forward extension of the SM is the addition of a fourth generation of fermions. While measurements at LEP and in the flavor sector set stringent limits on the parameters of such an extension, it is not fully excluded yet $70 / 71 / 72 \mid 73$. The measurement of the $Z$ lineshape, for example, implies that the fourth generation neutrino has a mass exceeding $46.7 \mathrm{GeV} / c^{2}$ 74. An intriguing feature of a fourth generation of quarks is the possibility to allow for large $\mathrm{CP}$ violation effects that would be big enough to explain the baryon asymmetry in the universe 75 .

The CDF collaboration has used the $t \bar{t}$ candidate sample of lepton+jets events to search for a fourth-generation up-type quark $t^{\prime}$ and sets a lower limit of $m_{t^{\prime}}>$ $335 \mathrm{GeV} / c^{2}$ at the $95 \%$ C.L. $\frac{76}{76}$ with collision data corresponding to $4.6 \mathrm{pb}^{-1}$. Another analysis searched for the pair production of heavy fourth-generation downtype quarks in the decay channel $b^{\prime} \bar{b}^{\prime} \rightarrow t W^{-} \bar{t} W^{+}$by looking for events with two same-charge leptons $\left(e\right.$ or $\mu$ ), several jets and $E_{\mathrm{T}}$. No significant excess of such events is found and a lower limit of $m_{b^{\prime}}>338 \mathrm{GeV} / c^{2}$ at the $95 \%$ C.L. is set 77 .

\section{Top-Quark Decay Properties}

Within the SM, top quarks decay predominantly into a $b$ quark and a $W$ boson, while the decays $t \rightarrow d+W^{+}$and $t \rightarrow s+W^{+}$are strongly CKM suppressed and can be neglected. The top-quark decay rate, including first order QCD corrections, is given by

$$
\Gamma_{t}=\frac{G_{F} m_{t}^{3}}{8 \pi \sqrt{2}}\left|V_{t b}\right|^{2}\left(1-\frac{M_{W}^{2}}{m_{t}^{2}}\right)^{2}\left(1+2 \frac{M_{W}^{2}}{m_{t}^{2}}\right)\left[1-\frac{2 \alpha_{s}}{3 \pi} \cdot f(y)\right]
$$

with $y=\left(M_{W} / m_{t}\right)^{2}$ and $f(y)=2 \pi^{2} / 3-2.5-3 y+4.5 y^{2}-3 y^{2} \ln y$ |78|79|80, yielding $\Gamma_{t}=1.34 \mathrm{GeV}$ at $m_{t}=172.6 \mathrm{GeV} / c^{2}$. The large $\Gamma_{t}$ implies a very short lifetime of $\tau_{t}=1 / \Gamma_{t} \approx 0.5 \cdot 10^{-24} \mathrm{~s}$ which is smaller than the characteristic formation time of hadrons $\tau_{\text {form }} \approx 1 \mathrm{fm} / c \approx 3 \cdot 10^{-24} \mathrm{~s}$. In other words, top quarks decay before they can couple to light quarks and form hadrons.

\subsection{W-Boson Helicity in Top-Quark Decays}

The amplitude of the decay $t \rightarrow b+W^{+}$is dominated by the contribution from longitudinal $W$ bosons because the decay rate of the longitudinal component scales 
with $m_{t}^{3}$, while the decay rate into transverse $W$ bosons increases only linearly with $m_{t}$. In both cases the $W^{+}$couples solely to $b$ quarks of left-handed chirality, which translates into left-handed helicity, since the $b$ quark is effectively massless compared to the energy scale set by $m_{t}$. If the $b$ quark is emitted antiparallel to the top-quark spin axis, the $W^{+}$must be longitudinally polarized, $h^{W}=0$, to conserve angular momentum. If the $b$ quark is emitted parallel to the top-quark spin axis, the $W^{+}$boson has helicity $h^{W}=-1$ and is transversely polarized. $W$ bosons with positive helicity are thus forbidden in top-quark decays due to angular momentum conservation, assuming $m_{b}=0$. The fraction of longitudinally polarized $W$ bosons is predicted to be $f_{0}=m_{t}^{2} /\left(2 m_{W}^{2}+m_{t}^{2}\right) \simeq 0.70$ and the left-handed fraction $f_{-}=2 m_{W}^{2} /\left(2 m_{W}^{2}+m_{t}^{2}\right) \simeq 0.30$. Taking the non-zero $b$-quark mass into account yields very small corrections, leading to a non-zero fraction of $W$ bosons in top-quark decay with positive helicity $f_{+}=3.6 \cdot 10^{-4}$ at Born level 81 .

The spin orientation (helicity) of $W$ bosons from top-quark decays is propagated to its decay products. In case of leptonic $W$ decays the polarization is preserved and can be measured. A useful observable for experiments is therefore the cosine of the polarization angle $\theta^{*}$ which is defined as the angle of the charged lepton in the $W$-boson decay frame measured with respect to the top-quark direction. The probability density $\omega$ has the following distribution:

$$
\begin{array}{r}
\omega\left(\theta^{*}\right)=f_{0} \cdot \omega_{0}\left(\theta^{*}\right)+f_{+} \cdot \omega_{+}\left(\theta^{*}\right)+\left(1-f_{0}-f_{+}\right) \cdot \omega_{-}\left(\theta^{*}\right) \text { with } \\
\omega_{0}\left(\theta^{*}\right)=\frac{3}{4}\left(1-\cos ^{2} \theta^{*}\right), \omega_{+}\left(\theta^{*}\right)=\frac{3}{8}\left(1+\cos \theta^{*}\right)^{2}, \omega_{-}\left(\theta^{*}\right)=\frac{3}{8}\left(1-\cos \theta^{*}\right)^{2} .
\end{array}
$$

Several measurements of the helicity fractions have been performed by the CDF and $\mathrm{D} \varnothing$ collaborations. One class of analyses reconstruct the $t \bar{t}$ event kinematics in lepton+jets events and measure $\cos \theta^{*}$ directly $22|83| 84|85| 86$, while a second class uses the square of the invariant mass of the lepton and the $b$-quark jet $M_{\ell b}^{2}$ in the

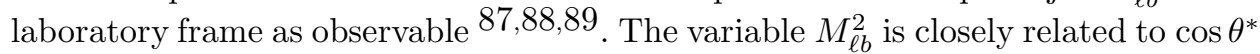
and has the advantage that it can also be used in $t \bar{t}$ dilepton events. In Fig. 6 the observed distribution of $\cos \theta^{*} \underline{86}$ after deconvoluting acceptance and reconstruction effects is compared to the probability densities $\omega$ of the three $W$ boson helicities scaled to the $t \bar{t}$ cross section.

A third set of analyses $\frac{8890}{19}$ uses the $p_{T}$ spectrum of the charged lepton which is also sensitive to the helicity fractions because the $V$ - $A$ structure of the $W$-boson decay causes a strong correlation between the helicity of the $W$ boson and the lepton momentum. Qualitatively, this can be understood as follows: The $\nu_{\ell}$ from the $W^{+}$ decay is always left-handed, the $\ell^{+}$is right-handed. In the case of a left-handed $W^{+}$ boson angular momentum conservation demands therefore that the $\ell^{+}$is emitted in the direction of the $W^{+}$spin, that means antiparallel to the $W^{+}$momentum. That is why charged leptons from the decay of left-handed $W$ bosons are softer than charged leptons from longitudinal $W$ bosons, which are mainly emitted in the direction transverse to the $W$ boson momentum. The spectrum of leptons from righthanded $W$ bosons would be even more harder than the one from longitudinal ones, 


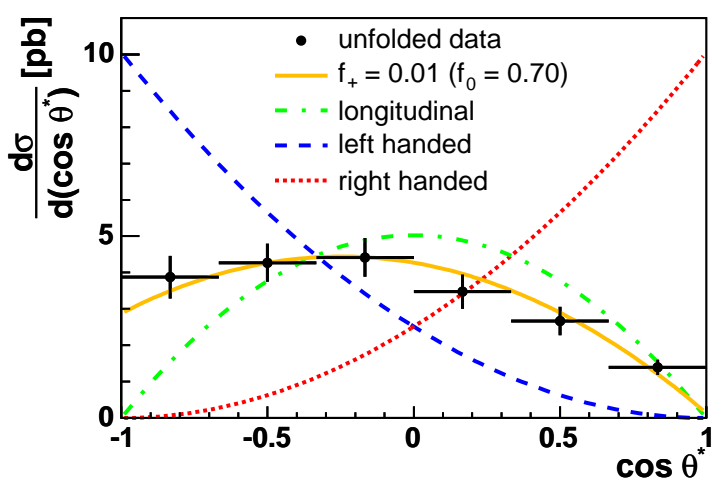

Fig. 6. The deconvoluted distribution of $\cos \theta^{*}$ normalized to the inclusive $t \bar{t}$ cross section is compared to theoretically predicted curves of purely left handed, right handed, and longitudinally polarized $W$ bosons from top-quark decay. The measurement by the CDF collaboration uses data corresponding to $1.9 \mathrm{fb}^{-1}$.

since they would be emitted preferentially in the direction of the $W$ momentum.

A fourth technique to measure the helicity fractions uses the LO matrix elements of $t \bar{t}$ production 919293 .

More recent analyses measure the fractions $f_{0}$ and $f_{+}$simultaneously in a twodimensional fit, while in previous analyses two fits were performed keeping one of the fractions at its SM value $85 / 86 / 93$. Using the matrix element technique CDF obtains $f_{0}=0.88 \pm 0.11$ (stat) \pm 0.06 (syst) and $f_{+}=-0.15 \pm 0.07$ (stat) \pm 0.06 (syst) with a correlation coefficient of $-59 \% 93$. The result is in good agreement with the SM prediction.

\subsection{Form Factors of the Wtb Vertex}

The D $\varnothing$ collaboration has performed a very general analysis of the $W t b$ vertex 94 . When including operators up to dimension five, the Dirac structure of the $W t b$ vertex can be generalized by the interaction Lagrangian

$$
\mathcal{L}=\frac{g_{w}}{\sqrt{2}}\left[W_{\mu}^{-} \bar{b} \gamma^{\mu}\left(f_{1}^{L} P_{-}+f_{1}^{R} P_{+}\right) t-\frac{1}{m_{W}} \partial_{\nu} W_{\mu}^{-} \bar{b} \sigma^{\mu \nu}\left(f_{2}^{L} P_{-}+f_{2}^{R} P_{+}\right) t\right]+\text { h.c. },
$$

where $P_{ \pm}=\frac{1}{2}\left(1 \pm \gamma^{5}\right)$ and $i \sigma^{\mu \nu}=-\frac{1}{2}\left[\gamma^{\mu}, \gamma^{\nu}\right]$ 95. The four form factors $f_{1,2}^{L, R}$ are assumed to be real numbers. In standard electroweak theory they take the values $f_{1}^{L}=1$ and $f_{1}^{R}=f_{2}^{L}=f_{2}^{R}=0$, such that the production of right-handed $W$ bosons from top-quark decay is suppressed. D $\varnothing$ pursues a general strategy to experimentally determine allowed regions of parameter space for all four form factors in Eq. 6. The experimental input is the distribution of $\cos \theta^{*}$ as observed in $t \bar{t}$ candidate events and the rate of single top-quark events 94 . 


\subsection{The measurement of $\mathcal{R}_{b}$}

In the SM the top quark is predicted to decay to a $W$ boson and a $b$ quark with a branching fraction $\mathcal{R}_{b} \equiv \mathrm{BR}(t \rightarrow W b)$ close to $100 \%$. This prediction is obtained in the following way. In general, the top quark can decay in three channels $t \rightarrow$ $d / s / b+W^{+}$and $\mathcal{R}_{b}$ is given by the ratio of the squares of the relevant CKM matrix elements: $\mathcal{R}_{b}=\left|V_{t b}\right|^{2} /\left(\left|V_{t d}\right|^{2}+\left|V_{t s}\right|^{2}+\left|V_{t b}\right|^{2}\right.$. In the SM the CKM matrix has to be unitary $\left(\mathbf{V V}^{\dagger}=\mathbf{V}^{\dagger} \mathbf{V}=\mathbf{1}\right.$ ), which leads to $\left|V_{t d}\right|^{2}+\left|V_{t s}\right|^{2}+\left|V_{t b}\right|^{2}=1$ and thereby to $\mathcal{R}_{b}=\left|V_{t b}\right|^{2}$. Our present knowledge on $\left|V_{t b}\right|$ stems primarily from measurements of $b$-meson and $c$-meson decays which determine the values of the other CKM matrix elements. Using the unitarity condition of the CKM matrix one can obtain $\left|V_{t b}\right|$ in an indirect way. This method yields $\left|V_{t b}\right|=0.999133 \pm 0.000044$ with very high precision 96 .

Only recently a determination of $\left|V_{t b}\right|$ without unitarity assumption was obtained from the measurement of the single top-quark cross section, yielding $\left|V_{t b}\right|=0.88 \pm$ 0.07 97. However, if a fourth generation of quarks was present, the unitarity of the $3 \times 3$ CKM matrix could be violated. Therefore, it is desirable to make a direct measurement of $\mathcal{R}_{b}$ using $t \bar{t}$ candidate events.

In most $t \bar{t}$ cross section analyses the assumption $\mathcal{R}_{b}=1$ is made, but CDF and $\mathrm{D} \varnothing$ have also made two measurements without this constraint 9899|100. In the latest analysis from $\mathrm{D} \varnothing 100$ the $W+$ jets data set is split in various disjoint subsets according to the number of jets $(0,1$, or $\geq 2)$, the charged lepton type (electron or muon), and most importantly the number of $b$-tagged jets. The fit results are: $\mathcal{R}_{b}=0.97_{-0.08}^{+0.09}$ and $\sigma(t \bar{t})=8.18_{-0.84}^{+0.90} \pm 0.50$ (lumi) pb, where the statistical and systematic uncertainties have been combined. The lower limit on $\mathcal{R}_{b}$ is determined to be $\mathcal{R}_{b}>0.79$ at the $95 \%$ C.L.

\subsection{Search for Non-SM Top-Quark Decays}

Two different classes of non-SM top-quark decays have been searched for at the Tevatron, decays via flavor-changing neutral currents (FCNC) and into a charged Higgs boson.

\subsubsection{FCNC-induced Top-Quark Decays}

In the SM FCNC are not present at tree level, but rather occur only through loop processes in higher orders of perturbation theory. In the top sector in particular FCNC are strongly suppressed with branching ratios of $\mathcal{O} \approx 10^{-14}$. The CDF collaboration has searched for non-SM top quark decays of the type $t \bar{t} \rightarrow$ $Z q W^{-} \bar{b} \rightarrow\left(\ell^{+} \ell^{-} q\right)\left(q \bar{q}^{\prime} \bar{b}\right)$. Top-antitop candidates are reconstructed in events with four high- $p_{\mathrm{T}}$ jets and two isolated leptons using kinematic constraints. Within their experimental resolutions determined from simulated events the reconstructed mass of the hadronically decaying $W$-boson $M_{q q}$ has to be equal to $m_{W}$, the mass of the reconstructed hadronic top quark $M_{b q q}$ and the mass of semileptonically de- 
caying top quark $M_{Z q}$ has to resemble $m_{t}$. A $\chi^{2}$ formed from these conditions is used to determine the most likely combination of physics objects and measure its $t \bar{t}$-likeness under the anomalous-decay hypothesis. A fit to the $\sqrt{\chi^{2}}$ distribution in combination with a Feldman-Cousins technique is used to derive an upper limit on the branching ratio: $\mathrm{BR}(t \rightarrow Z q)<3.7 \%$ at the $95 \%$ C.L. 101] A previous Run I result by $\mathrm{CDF}$ also set a limit on FCNC branching ratios in the photon-plus-jet mode: $\operatorname{BR}(t \rightarrow \gamma q)<3.2 \% 102$.

\subsubsection{Top-Quark Decays to a Charged Higgs Boson}

In the SM a single complex Higgs doublet scalar field is responsible for breaking the electroweak symmetry and generating the masses of gauge bosons and fermions. Many extensions of the SM include a Higgs sector with two Higgs doublets and are therefore called Two Higgs Doublet Models (THDM). In a THDM electroweak symmetry breaking leads to five physical Higgs bosons: two neutral $C P$-even scalars $h^{0}$ and $H^{0}$, one neutral $C P$-odd pseudoscalar $A^{0}$, and a pair of charged scalars $H^{ \pm}$. The parameters of the extended Higgs sector include the mass of the charged Higgs $M_{H^{+}}$and $\tan \beta=v_{1} / v_{2}$, the ratio of the vacuum expectation values $v_{1}$ and $v_{2}$ of the two Higgs doublets. The minimal supersymmetric model (MSSM) is an example of a THDM.

If the charged Higgs boson is lighter than the difference between top-quark mass and $b$-quark mass, $m_{H^{ \pm}}<m_{t}-m_{b}$, the decay mode $t \rightarrow H^{+} b$ is possible and competes with the SM decay $t \rightarrow W^{+} b$. The branching fraction depends on $\tan \beta$ and $m_{H^{+}}$. The MSSM predicts that the channel $t \rightarrow H^{+} b$ dominates the top-quark decay for $\tan \beta \gtrsim 70$. In most analyses it is assumed that $\mathrm{BR}\left(t \rightarrow W^{+} b\right)+\mathrm{BR}(t \rightarrow$ $\left.H^{+} b\right)=1$. In the parameter region $\tan \beta<1$ the dominant decay mode is $H^{+} \rightarrow c \bar{s}$, while for $\tan \beta>1$ the decay channel $H^{+} \rightarrow \tau^{+} \nu_{\tau}$ is the most important one. For $\tan \beta>5$ the branching fraction to $\tau^{+} \nu_{\tau}$ is nearly $100 \%$. Thus, in this region of parameter space THDM models predict an excess of $t \bar{t}$ events with tau leptons over the SM expectation.

First searches for the $H^{ \pm}$in top-quark events were already performed well before the top quark was discovered in 1994/95. The UA1 and UA2 experiments at the CERN $S p \bar{p} S$ excluded certain regions of the $m_{t}$ versus $m_{H^{ \pm}}$plane 1031104 . In Run I of the Tevatron, CDF and D $\varnothing$ improved these limits using events with a dilepton signature 105 or reconstructing tau leptons in their hadronic decay mode 106/107/108/109/110, which is experimentally a very challenging task at a hadron collider. In Tevatron Run II, CDF has added a direct search for a charged Higgs boson in the decay channel $H^{+} \rightarrow c \bar{s}$ using the dijet invariant mass as a discriminant 111. In a reanalysis of the $t \bar{t}$ candidate samples used for cross section measurements CDF also considered the decay modes $H^{+} \rightarrow t^{*} \bar{b}$ and $H^{+} \rightarrow W^{+} h^{0}$ with $h^{0} \rightarrow b \bar{b} 112$. The $\mathrm{D} \varnothing$ collaboration has recently performed direct searches in the lepton+jets 113 and dilepton channels 114 , but has also done a simultaneous measurement of the $t \bar{t}$ cross section in the lepton+jets, the dilepton and the 
$\tau+$ lepton channel, deriving in parallel limits on top-quark decays to a charged Higgs boson 44 . So far, there is no evidence for $t \rightarrow H^{+} b$ decays. The resulting limits on the branching ratio $\mathrm{BR}\left(t \rightarrow H^{+} b\right)$ depend on $m_{H^{ \pm}}$and assumptions made on the Higgs decays modes, but are typically in the range of $15 \%$ to $25 \%$.

\section{Conclusions}

Large data sets of $t \bar{t}$ candidate events are now available to the Tevatron collaborations $\mathrm{CDF}$ and $\mathrm{D} \varnothing$ and facilitate precise investigations of top-quark properties. The $t \bar{t}$ production cross section has been measured with a relative precision of $6.5 \%$ and is in excellent agreement with QCD predictions 48. Many interesting analyses have searched for physics beyond the SM, for example for resonances decaying into $t \bar{t}$ pairs. The measured forward-backward asymmetry in $t \bar{t}$ production shows an intriguing excess which will come under closer scrutiny once more data will be analysed. The measurements of top-quark decay show impressive progress, for example the measurement of the $W$-helicity fractions in top decay. More detailed reviews on top-quark physics are available on phenomenology 115, Tevatron Run I results 116/117/118|119, and Tevatron Run II results 120/121|122|123/124|125.

\section{Acknowledgments}

The author would like to thank his colleagues Frederic Deliot, Fabrizio Margaroli, and Tom Schwarz as well as Werner Bernreuther for useful discussions on the manuscript and acknowledges the financial support of the Helmholtz-Alliance Physics at the Terascale.

\section{References}

1. [Tevatron Electroweak Working Group and CDF Collaboration and D0 Collab], arXiv:0903.2503 [hep-ex].

2. R.D. Peccei, S. Peris, and X. Zhang, Nucl. Phys. B 349 (1991) 305-322.

3. D. E. Acosta et al. [CDF Collaboration], Phys. Rev. D 71 (2005) 052003 arXiv:hep-ex/0410041.

4. D. E. Acosta et al. [CDF-II Collaboration], Phys. Rev. D 71 (2005) 072005 arXiv:hep-ex/0409029.

5. A. Abulencia et al. [CDF Collaboration], Phys. Rev. Lett. 97 (2006) 082004 arXiv:hep-ex/0606017.

6. V. M. Abazov et al. [D0 Collaboration], Phys. Rev. D 74 (2006) 112004 arXiv:hep-ex/0611002.

7. V. M. Abazov et al. [D0 Collaboration], Phys. Lett. B 626 (2005) 35 arXiv:hep-ex/0504058.

8. A. Abulencia et al. [CDF Collaboration and CDF - Run II Collaboration], Phys. Rev. D 74 (2006) 072006 arXiv:hep-ex/0607035.

9. D. E. Acosta et al. [CDF Collaboration], Phys. Rev. D 72 (2005) 032002 arXiv:hep-ex/0506001.

10. T. Aaltonen et al. [CDF Collaboration], Phys. Rev. D 79 (2009) 052007 arXiv:0901.4142 [hep-ex]]. 
11. V. M. Abazov et al. [D0 Collaboration], Phys. Rev. Lett. 100 (2008) 192004 arXiv:0803.2779 [hep-ex]].

12. T. Aaltonen et al. [CDF Collaboration], public conf. note no. 10049, January 2010.

13. T. Aaltonen et al. [CDF Collaboration], arXiv:1004.3224 [hep-ex].

14. M. L. Mangano, M. Moretti, F. Piccinini, R. Pittau and A. D. Polosa, JHEP 0307 (2003) 001 arXiv:hep-ph/0206293.

15. S. Richter, Ph.D. thesis (University of Karlsruhe), FERMILAB-THESIS-2007-35 (2007).

16. M. Renz, Master thesis (University of Karlsruhe), FERMILAB-MASTERS-2008-06 (2008).

17. J. Lueck, Ph.D. thesis (University of Karlsruhe), FERMILAB-THESIS-2009-33 (2009).

18. V. M. Abazov et al. [D0 Collaboration], Phys. Lett. B 626 (2005) 45 arXiv:hep-ex/0504043.

19. D. E. Acosta et al. [CDF Collaboration], Phys. Rev. D 72 (2005) 052003 arXiv:hep-ex/0504053.

20. V. M. Abazov et al. [D0 Collaboration], Phys. Rev. D 76 (2007) 092007 arXiv:0705.2788 [hep-ex]].

21. T. Aaltonen et al. (CDF Collaboration), public conf. note no. 9474, August 2009.

22. F. Abe et al. [CDF Collaboration], Phys. Rev. D 50 (1994) 2966.

23. F. Abe et al. [CDF Collaboration], Phys. Rev. Lett. 74 (1995) 2626 arXiv:hep-ex/9503002.

24. S. Abachi et al. [D0 Collaboration], Phys. Rev. Lett. 74 (1995) 2632 arXiv:hep-ex/9503003.

25. D. E. Acosta et al. [CDF Collaboration], Phys. Rev. Lett. 93 (2004) 142001 arXiv:hep-ex/0404036.

26. A. Abulencia et al. [CDF Collaboration], Phys. Rev. D 78 (2008) 012003 arXiv:hep-ex/0612058.

27. T. Aaltonen et al. [CDF Collaboration], Phys. Rev. D 79 (2009) 112007 arXiv:0903.5263 [hep-ex]].

28. T. Aaltonen et al. [CDF Collaboration], public conf. note no. 9890, August 2009.

29. V. M. Abazov et al. [D0 Collaboration], Phys. Lett. B 626 (2005) 55 arXiv:hep-ex/0505082.

30. V. M. Abazov et al. [D0 Collaboration], Phys. Rev. D 76 (2007) 052006 arXiv:0706.0458 [hep-ex]].

31. V. M. Abazov et al. [D0 Collaboration], Phys. Lett. B 679 (2009) 177 arXiv:0901.2137 [hep-ex]].

32. V. M. Abazov et al. [D0 Collaboration], public conf. note no. 6038, March 2010.

33. F. Abe et al. [CDF Collaboration], Phys. Rev. Lett. 79 (1997) 1992.

34. B. Abbott et al. [D0 Collaboration], Phys. Rev. Lett. 83 (1999) 1908 arXiv:hep-ex/9901023.

35. A. Abulencia et al. [CDF - Run II Collaboration], Phys. Rev. D 74 (2006) 072005 arXiv:hep-ex/0607095.

36. T. Aaltonen et al. [CDF Collaboration], Phys. Rev. D 76 (2007) 072009 arXiv:0706.3790 [hep-ex]].

37. T. Aaltonen et al. [The CDF Collaboration], arXiv:1002.0365 [hep-ex]. Accepted by Phys. Rev. D.

38. V. M. Abazov et al. [D0 Collaboration], Phys. Rev. D 76 (2007) 072007 arXiv:hep-ex/0612040.

39. V. M. Abazov et al. [D0 Collaboration], arXiv:0911.4286 [hep-ex]. 


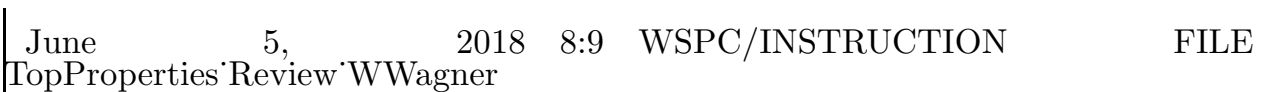

Top-Quark Cross Section and Properties at the Tevatron

40. A. A. Affolder et al. [CDF Collaboration], Phys. Rev. D 64 (2001) 032002 [Erratumibid. D 67 (2003) 119901] arXiv:hep-ex/0101036.

41. V. M. Abazov et al. [D0 Collaboration], Phys. Rev. D 67 (2003) 012004 arXiv:hep-ex/0205019.

42. N. Kidonakis and R. Vogt, Phys. Rev. D 68 (2003) 114014 arXiv:hep-ph/0308222.

43. T. Aaltonen et al. [CDF Collaboration], public conf. note no. 9913, October 2009.

44. V. M. Abazov et al. [D0 Collaboration], Phys. Rev. D 80 (2009) 071102 arXiv:0903.5525 [hep-ex]].

45. M. Cacciari, S. Frixione, M. L. Mangano, P. Nason and G. Ridolfi, JHEP 0809 (2008) 127 arXiv:0804.2800 [hep-ph]].

46. N. Kidonakis and R. Vogt, Phys. Rev. D 78 (2008) 074005 arXiv:0805.3844 [hep-ph]].

47. S. Moch and P. Uwer, Phys. Rev. D 78 (2008) 034003 [arXiv:0804.1476 [hep-ph]].

48. S. Moch and P. Uwer, Nucl. Phys. Proc. Suppl. 183 (2008) 75 arXiv:0807.2794 [hep$\mathrm{ph}]$.

49. LEP and Tevatron Electroweak Working Groups, CERN-PH-EP/2009-023, FERMILAB-TM-2446-E, November 2009.

50. T. Aaltonen et al. [CDF Collaboration], Phys. Rev. D 78, 111101 (2008) arXiv:0712.3273 [hep-ex]].

51. T. Aaltonen et al. [CDF Collaboration], Phys. Rev. D 79, 031101 (2009) arXiv:0807.4262 [hep-ex]].

52. T. Aaltonen et al. [CDF Collaboration], CDF public note no. 9432, July 2008.

53. T. Aaltonen et al. [CDF Collaboration], CDF public note no. 10048, January 2010.

54. W. Bernreuther, A. Brandenburg, Z. G. Si and P. Uwer, Phys. Rev. Lett. 87 (2001) 242002 arXiv:hep-ph/0107086].

55. W. Bernreuther, A. Brandenburg, Z. G. Si and P. Uwer, Nucl. Phys. B 690 (2004) 81 arXiv:hep-ph/0403035.

56. J. H. Kuhn and G. Rodrigo, Phys. Rev. D 59 (1999) 054017 arXiv:hep-ph/9807420.

57. O. Antunano, J. H. Kuhn and G. Rodrigo, Phys. Rev. D 77 (2008) 014003 arXiv:0709.1652 [hep-ph]].

58. W. Bernreuther and Z. G. Si, arXiv:1003.3926 [hep-ph].

59. S. Dittmaier, P. Uwer and S. Weinzierl, Phys. Rev. Lett. 98 (2007) 262002 arXiv:hep-ph/0703120.

60. L. G. Almeida, G. Sterman and W. Vogelsang, Phys. Rev. D 78 (2008) 014008 arXiv:0805.1885 [hep-ph]].

61. J. L. Rosner, Phys. Lett. B 387 (1996) 113 arXiv:hep-ph/9607207.

62. T. Aaltonen et al. [CDF Collaboration], public conf. note no. 9724, March 2009.

63. V.M. Abazov et al. [DØ Collaboration], Phys. Rev. Lett. 100, 142002 (2008).

64. T. Aaltonen et al. [CDF Collaboration], Phys. Rev. Lett. 101, 202001 (2008) arXiv:0806.2472 [hep-ex]].

65. V. M. Abazov et al. [D0 Collaboration], Phys. Rev. Lett. 92 (2004) 221801 arXiv:hep-ex/0307079.

66. T. Aaltonen et al. [CDF Collaboration], Phys. Rev. D 77 (2008) 051102 arXiv:0710.5335 [hep-ex]].

67. V.M. Abazov et al. [DØ Collaboration], public conf. note no. 5882 (2009).

68. T. Aaltonen et al. [CDF Collaboration], public conf. note no. 9844, July 2009.

69. T. Aaltonen et al. [CDF Collaboration], arXiv:0911.3112 [hep-ex].

70. G. D. Kribs, T. Plehn, M. Spannowsky and T. M. P. Tait, Phys. Rev. D 76 (2007) 075016 arXiv:0706.3718 [hep-ph]].

71. P. Q. Hung and M. Sher, Phys. Rev. D 77 (2008) 037302 arXiv:0711.4353 [hep-ph]].

72. M. Bobrowski, A. Lenz, J. Riedl and J. Rohrwild, Phys. Rev. D 79 (2009) 113006 
arXiv:0902.4883 [hep-ph]].

73. A. J. Buras, B. Duling, T. Feldmann, T. Heidsieck, C. Promberger and S. Recksiegel, arXiv:1002.2126 [hep-ph].

74. S. S. Bulanov, V. A. Novikov, L. B. Okun, A. N. Rozanov and M. I. Vysotsky, Phys. Atom. Nucl. 66 (2003) 2169 [Yad. Fiz. 66 (2003) 2219] arXiv:hep-ph/0301268].

75. W. S. Hou, Chin. J. Phys. 47 (2009) 134 [arXiv:0803.1234 [hep-ph]].

76. T. Aaltonen et al. [CDF Collaboration], public conf. note no. 10110, March 2010.

77. T. Aaltonen et al. [The CDF Collaboration], arXiv:0912.1057 [hep-ex].

78. M. Jezabek and J. H. Kuhn, Phys. Lett. B 207 (1988) 91.

79. M. Jezabek and J. H. Kuhn, Nucl. Phys. B 314 (1989) 1.

80. J. H. Kuhn, arXiv:hep-ph/9707321

81. M. Fischer, S. Groote, J. G. Korner and M. C. Mauser, Phys. Rev. D 63 (2001) 031501 arXiv:hep-ph/0011075.

82. V. M. Abazov et al. [D0 Collaboration], Phys. Rev. D 72 (2005) 011104 arXiv:hep-ex/0505031.

83. V. M. Abazov et al. [D0 Collaboration], Phys. Rev. D 75 (2007) 031102 arXiv:hep-ex/0609045.

84. A. Abulencia et al. [CDF II Collaboration], Phys. Rev. D 75 (2007) 052001 arXiv:hep-ex/0612011.

85. V. M. Abazov et al. [D0 Collaboration], Phys. Rev. Lett. 100 (2008) 062004 arXiv:0711.0032 [hep-ex]].

86. T. Aaltonen et al. [CDF Collaboration], Phys. Lett. B 674, 160 (2009) arXiv:0811.0344 [hep-ex]].

87. D. E. Acosta et al. [CDF Collaboration], Phys. Rev. D 71 (2005) 031101 [Erratumibid. D 71 (2005) 059901] arXiv:hep-ex/0411070.

88. A. Abulencia et al. [CDF Collaboration], Phys. Rev. D 73 (2006) 111103 arXiv:hep-ex/0511023.

89. A. Abulencia et al. [CDF Collaboration], Phys. Rev. Lett. 98 (2007) 072001 arXiv:hep-ex/0608062.

90. A. A. Affolder et al. [CDF Collaboration], Phys. Rev. Lett. 84 (2000) 216 arXiv:hep-ex/9909042.

91. V. M. Abazov et al. [D0 Collaboration], Phys. Lett. B 617 (2005) 1 arXiv:hep-ex/0404040.

92. T. Aaltonen et al. [CDF Collaboration], public conf. note no. 9144, November 2007.

93. T. Aaltonen et al. [The CDF Collaboration], arXiv:1003.0224] [hep-ex].

94. V. M. Abazov et al. [D0 Collaboration], Phys. Rev. Lett. 102 (2009) 092002 arXiv:0901.0151 [hep-ex]].

95. G. L. Kane, G. A. Ladinsky and C. P. Yuan, Phys. Rev. D 45 (1992) 124.

96. C. Amsler et al. [Particle Data Group], Phys. Lett. B 667 (2008) 1.

97. T. E. W. Group [CDF Collaboration and D0 Collaboration], arXiv:0908.2171 [hep-ex].

98. A. A. Affolder et al. [CDF Collaboration], Phys. Rev. Lett. 86 (2001) 3233 arXiv:hep-ex/0012029.

99. D. E. Acosta et al. [CDF Collaboration], Phys. Rev. Lett. 95 (2005) 102002 arXiv:hep-ex/0505091.

100. V. M. Abazov et al. [D0 Collaboration], Phys. Rev. Lett. 100 (2008) 192003 arXiv:0801.1326 [hep-ex]].

101. T. Aaltonen et al. [CDF Collaboration], Phys. Rev. Lett. 101 (2008) 192002 arXiv:0805.2109 [hep-ex]].

102. F. Abe et al. [CDF Collaboration], Phys. Rev. Lett. 80 (1998) 2525.

103. C. Albajar et al. [UA1 Collaboration], Phys. Lett. B 257 (1991) 459. 


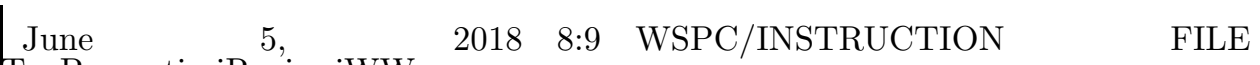

TopProperties`Review`WWagner

Top-Quark Cross Section and Properties at the Tevatron

104. J. Alitti et al. [UA2 Collaboration], Phys. Lett. B 280 (1992) 137.

105. F. Abe et al. [CDF Collaboration], Phys. Rev. Lett. 73 (1994) 2667.

106. F. Abe et al. [CDF Collaboration], Phys. Rev. Lett. 72 (1994) 1977.

107. F. Abe et al. [CDF Collaboration], Phys. Rev. D 54 (1996) 735 arXiv:hep-ex/9601003.

108. F. Abe et al. [CDF Collaboration], Phys. Rev. Lett. 79 (1997) 357 arXiv:hep-ex/9704003.

109. A. A. Affolder et al. [CDF Collaboration], Phys. Rev. D 62 (2000) 012004 arXiv:hep-ex/9912013.

110. V. M. Abazov et al. [D0 Collaboration], Phys. Rev. Lett. 88 (2002) 151803 arXiv:hep-ex/0102039.

111. T. Aaltonen et al. [CDF Collaboration], Phys. Rev. Lett. 103 (2009) 101803 arXiv:0907.1269 [hep-ex]].

112. A. Abulencia et al. [CDF Collaboration], Phys. Rev. Lett. 96 (2006) 042003 arXiv:hep-ex/0510065.

113. V. M. Abazov et al. [D0 Collaboration], Phys. Rev. D 80 (2009) 051107 arXiv:0906.5326 [hep-ex]].

114. V. M. Abazov et al. [D0 Collaboration], Phys. Lett. B 682 (2009) 278 arXiv:0908.1811 [hep-ex]].

115. W. Bernreuther, J. Phys. G 35 (2008) 083001 arXiv:0805.1333 [hep-ph]].

116. C. Campagnari and M. Franklin, Rev. Mod. Phys. 69 (1997) 137 arXiv:hep-ex/9608003.

117. P. C. Bhat, H. Prosper and S. S. Snyder, Int. J. Mod. Phys. A 13 (1998) 5113 arXiv:hep-ex/9809011.

118. K. Tollefson and E. W. Varnes, Ann. Rev. Nucl. Part. Sci. 49 (1999) 435.

119. D. Chakraborty, J. Konigsberg and D. L. Rainwater, Ann. Rev. Nucl. Part. Sci. 53 (2003) 301 arXiv:hep-ph/0303092.

120. W. Wagner, Rept. Prog. Phys. 68 (2005) 2409 arXiv:hep-ph/0507207.

121. A. Quadt, Eur. Phys. J. C 48 (2006) 835.

122. R. Kehoe, M. Narain and A. Kumar, Int. J. Mod. Phys. A 23 (2008) 353 arXiv:0712.2733 [hep-ex]].

123. M. A. Pleier, Int. J. Mod. Phys. A 24 (2009) 2899 arXiv:0810.5226 [hep-ex]].

124. J. R. Incandela, A. Quadt, W. Wagner and D. Wicke, Prog. Part. Nucl. Phys. 63 (2009) 239 arXiv:0904.2499 [hep-ex]].

125. A. P. Heinson [CDF and D0 Collaboration], Mod. Phys. Lett. A 25 (2010) 309 arXiv:1002.4167 [hep-ex]]. 